\title{
Specific Genetic Deficiencies of the A and B Isoenzymes of Monoamine Oxidase Are Characterized by Distinct Neurochemical and Clinical Phenotypes
}

\author{
Jacques W.M. Lenders, ${ }^{\star}$ Graeme Eisenhofer, ${ }^{\ddagger}$ Nico G.G.M. Abeling, ${ }^{\S}$ Wolfgang Berger, $\|$ Dennis L. Murphy, ${ }^{\rrbracket}$ C. Henk Konings, ${ }^{\star *}$ \\ Liesbeth M. Bleeker Wagemakers, ${ }^{\ddagger}$ Irwin J. Kopin, ${ }^{\ddagger}$ Farouk Karoum, ${ }^{\S}$ Albert H. van Gennip, ${ }^{\S}$ and Han G. Brunnerl \\ *Department of Medicine, Division of General Internal Medicine and ${ }^{\|}$Department of Human Genetics, St. Radboud University Hospital, \\ 6525 GA Nijmegen, The Netherlands; ${ }^{\S}$ Departments of Pediatrics and Clinical Chemistry, Academic Medical Center, University of \\ Amsterdam, 1105 AZ Amsterdam, The Netherlands; ${ }^{\ddagger}$ Clinical Neuroscience Branch, National Institute of Neurological Disorders and \\ Stroke, and "Laboratory of Clinical Science, National Institute of Mental Health, National Institutes of Health, Bethesda, Maryland 20892; \\ ${ }^{\S}$ Neuroscience Center, St. Elizabeth's Hospital, National Institute of Mental Health, Washington, DC 20032; **Department of Clinical \\ Chemistry, Free University Hospital, 1081 HV Amsterdam; and $\$$ The Ophthalmic Research Institute, Department of Ophthalmogenetics, \\ University of Amsterdam, 1105 AZ Amsterdam, The Netherlands
}

\begin{abstract}
Monoamine oxidase (MAO) exists as two isoenzymes and plays a central role in the metabolism of monoamine neurotransmitters. In this study we compared the neurochemical phenotypes of previously described subjects with genetically determined selective lack of MAO-A or a lack of both MAO-A and MAO-B with those of two subjects with a previously described $X$ chromosome microdeletion in whom we now demonstrate selective MAO-B deficiency. Mapping of the distal deletion breakpoint demonstrates its location in intron 5 of the MAO-B gene, with the deletion extending proximally into the Norrie disease gene. In contrast to the borderline mental retardation and abnormal behavioral phenotype in subjects with selective MAO-A deficiency and the severe mental retardation in patients with combined MAO-A/MAO-B deficiency and Norrie disease, the MAOB-deficient subjects exhibit neither abnormal behavior nor mental retardation. Distinct neurochemical profiles characterize the three groups of MAO-deficient patients. In MAOA-deficient subjects, there is a marked decrease in deaminated catecholamine metabolites and a concomitant marked elevation of O-methylated amine metabolites. These neurochemical changes are only slightly exaggerated in patients with combined lack of MAO-A and MAO-B. In contrast, the only biochemical abnormalities detected in subjects with the MAO-B gene deletion are a complete absence of platelet MAO-B activity and an increased urinary excretion of phenylethylamine. The differences in neurochemical profiles indicate that, under normal conditions, MAO-A is considerably more important than MAO-B in the metabolism of biogenic amines, a factor likely to contribute to the different clinical phenotypes. (J. Clin. Invest. 1996. 97:1010-1019.) Key words: catecholamines $\cdot$ Norrie disease $\cdot$ metabolism • metanephrines • dihydroxyphenylglycol
\end{abstract}

Address correspondence to Dr. Jacques W.M. Lenders, Department of Medicine, Division of General Internal Medicine, St. Radboud University Hospital, Geert Grooteplein Zuid 8, 6525 GA Nijmegen, The Netherlands. Phone: 31-24-3614782; FAX: 31-24-3541734; E-mail: inwg_j1@AZNVX1.AZN.NL

Received for publication 28 July 1995 and accepted in revised form 29 November 1995.

J. Clin. Invest.

(C) The American Society for Clinical Investigation, Inc.

0021-9738/96/02/1010/10 \$2.00

Volume 97, Number 4, February 1996, 1010-1019

\section{Introduction}

Monoamine oxidase (MAO) ${ }^{1}$ an enzyme that plays a crucial role in the metabolic degradation of biogenic amines, exists in two functional forms: MAO-A and MAO-B (1-3). The two isoenzymes have different substrate and inhibitor specificities and are encoded by two genes on the short arm of the X chromosome (4).

Combined loss of MAO-A and MAO-B activity (MAO$\mathrm{AB})$ has been described in some patients with an $\mathrm{X}$ chromosome deletion that includes not only the Norrie disease gene but also the MAO-A and MAO-B genes (5-9). In addition to the blindness, hearing loss, and variable mental retardation that characterize Norrie disease (10), patients with the additional deletion of both MAO genes show profound mental retardation, autistic-like behavior, atonic seizures, altered peripheral autonomic function, and profound alterations in biogenic amine metabolism $(5,6,8)$. More recently, selective loss of MAO-A activity due to a point mutation in exon 8 of the MAO-A gene was described in a Dutch kindred $(11,12)$. In this kindred, selective MAO-A deficiency was associated with a clinical phenotype characterized by borderline mental retardation and impaired impulse control, including a propensity towards stress-induced aggression.

Understanding the consequences of genetic deficiencies of MAO isoenzymes requires an understanding of the role of these enzymes in the metabolism of biogenic amines. In addition to inactivating catecholamines and their O-methylated metabolites, MAO deaminates other biogenic amines including 5-hydroxytryptamine (5-HT) and the trace amines phenylethylamine, $m$-tyramine, and $p$-tyramine (13). The catecholamines and their deaminated metabolites are also subject to O-methylation by catechol- $O$-methyltransferase (COMT). MAO and COMT, in combination with alcohol dehydrogenase, are responsible for the production of a wide range of catecholamine metabolites, which for the purpose of this study can be divided into two groups: deaminated metabolites and O-methylated amine metabolites (Fig. 1).

The redundant nature of catecholamine metabolism enables one pathway to compensate for blockade of the other, so

1. Abbreviations used in this paper: COMT, catechol- $O$-methyl transferase; DA, dopamine; DHPG, dihydroxyphenylglycol; DOPA, dihydroxyphenylalanine; DOPAC, dihydroxyphenylacetic acid; EPI, epinephrine; 5-HT, 5-hydroxytryptamine; HVA, homovanillic acid; MAO, monoamine oxidase; MHPG, methoxyhydroxyphenylglycol; VMA, vanillylmandelic acid. 


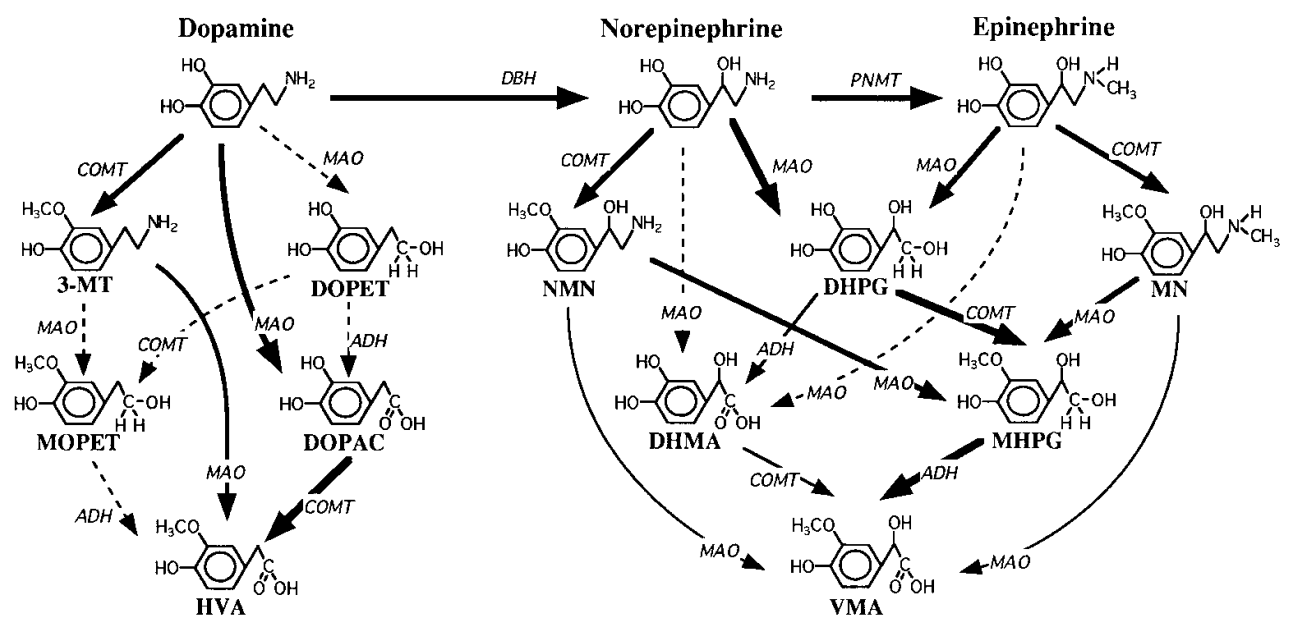

Figure 1. The metabolic pathways of degradation of NE, EPI, and DA. The deaminated metabolites are DHPG, DOPAC, dihydroxymandelic acid (DHMA), dihydroxy-phenylethanol (DOPET), methoxyhydroxyphenylethanol (MOPET), HVA, MHPG, and VMA. The O-methylated amine metabolites are normetanephrine $(N M N)$, metanephrine $(M N)$, and 3-MT. $D B H$, DA $\beta$-hydroxylase; $P N M T$, phenylethanolamine- $N$ methyltransferase; $A D H$, alcohol dehydrogenase. that reduced or absent activity of MAO leads to a decrease in production of deaminated metabolites and an increase in that of O-methylated amine metabolites $(14,15)$. Thus, simultaneous measurements of these metabolites are useful for neurochemical phenotyping of patients with disorders of catecholamine metabolism (16).

The relative roles of COMT and MAO in the metabolic degradation of catecholamines are well established; on the other hand, those of the isoenzymes of MAO are not clear but are important for understanding the basis of therapeutic interventions involving specific inhibition of one or the other of the two MAO isoenzymes. Additionally, establishing the relative roles of MAO-A and MAO-B may also provide insight into how neurochemical alterations associated with deficiencies of the two isoenzymes give rise to clinical syndromes.

In the present study, we report on two subjects with a hitherto undescribed selective deficiency of MAO-B due to a microdeletion of the $\mathrm{X}$ chromosome, extending from the Norrie disease gene to within the MAO-B gene (17). For comparison, the neurochemical characteristics of previously described patients with combined MAO-AB deficiency and selective MAO-A deficiency are reported in greater detail, including a comprehensive analysis of plasma catecholamine metabolites (Fig. 1). These comparisons enabled examination of the relative roles of MAO-A and MAO-B in the metabolic degradation of catecholamines and other biogenic amines, including 5-HT and the trace amines.

\section{Methods}

Subjects. Two brothers (ages: 29 and $31 \mathrm{yr}$ ) with a complete selective loss of MAO-B activity due to a microdeletion of the Norrie disease and MAO-B genes participated in the study. Norrie disease, characterized by blindness, was diagnosed at the ages of 5 and 18 mo. Progressive hearing loss due to cochlear degeneration was noticed in adolescence in these subjects. Two male members (ages: 24 and $34 \mathrm{yr}$ ) of an unrelated Dutch family with a complete selective deficiency of MAO-A were also included in this study. Affected males in this family have a point mutation of the MAO-A gene and are characterized clinically by borderline mental retardation and impaired impulse control $(11,12)$. In addition, the study included five male subjects (age: 12-23 yr) from three different families, with a microdeletion of the $\mathrm{X}$ chromosome, including the Norrie disease gene and the MAO-A and MAO-B genes (5-9). These latter subjects are characterized clinically by blindness and profound mental retardation.
29 males aged $21-55 \mathrm{yr}($ mean \pm SD: $35 \pm 9 \mathrm{yr}$ ) served as a reference group for measurements of plasma catecholamines and metabolites. Subjects were not taking any medications and were all in good health as determined by a negative medical history and a normal physical examination. These subjects were enlisted for study at two different institutions, The Clinical Center, National Institutes of Health (NIH), and the St. Radboud University Hospital. All procedures were approved by the appropriate review committees, and informed consent was obtained for all studies.

Procedures. Baseline venous blood samples were drawn after 15 min of rest, and plasma and platelet-rich plasma were separated by centrifugation at $4^{\circ} \mathrm{C}$. Samples of plasma and platelet-rich plasma were stored at $-80^{\circ} \mathrm{C}$ until assayed for concentrations of catecholamines, metabolites, and 5-HT (8), and activities of platelet MAO-B and plasma amine oxidase. 24-h urine samples were collected and acidified to $\mathrm{pH} 2-3$, and $20-\mathrm{ml}$ aliquots were stored at $-25^{\circ} \mathrm{C}$ until assayed. The two subjects with selective MAO-A deficiency received a simultaneous infusion of ${ }^{3} \mathrm{H}$-labeled $\mathrm{NE}$ and epinephrine (EPI) (L-2,5,6-[ $\left[{ }^{3} \mathrm{H}\right] \mathrm{NE}, \quad 40-60 \mathrm{Ci} / \mathrm{mmol} ; \quad$ L- $N$-methyl- $\left[{ }^{3} \mathrm{H}\right] \mathrm{EPI}, 65-75 \mathrm{Ci} /$ mmol [DuPont-New England Nuclear, 's Hertogenbosch, The Netherlands]) to calculate forearm spillovers of NE and normetanephrine (18). In these two subjects, a standardized tyramine infusion was also carried out to determine tyramine pressor sensitivity (19). To compare neurochemical abnormalities in MAO-deficient subjects with changes occurring after pharmacological inhibition of MAO, 11 male subjects (mean \pm SD ages: $37 \pm 9 \mathrm{yr}$ ) with attention deficit hyperactivity disorder were studied before and after administration of deprenyl at a dose (60 mg daily for $6 \mathrm{wk}$ ) sufficient to inhibit both MAO-A and MAO-B (19).

DNA/genetic analysis. Genomic DNA in the two MAO-B-deficient subjects was isolated from peripheral blood as described previously (20). DNA was digested by the restriction enzyme EcoRI, transferred to nylon membranes by Southern blotting, and hybridized to either ${ }^{32} \mathrm{P}$-labeled cosmid C5 (17) or to an MAO-B cDNA probe containing exons 3-15 (21). Hybridizing bands were visualized by overnight exposure of the membrane to film (X-omat S; Eastman Kodak Co., Rochester, NY). Hybridizing fragments were assigned to specific MAO-B exons based on published data (22). The detailed description of the genetic defects in the MAO-A- and MAO-AB-deficient subjects have been reported previously $(5-8,10-12)$.

Enzyme activities and platelet 5-HT. Platelet-rich plasma was prepared by differential centrifugation using EDTA or acid citrate dextrose as anticoagulants. Platelet MAO-B and plasma amine oxidase activities were measured at the NIH, using $200 \mu \mathrm{M}$ methylene$\left[{ }^{14} \mathrm{C}\right]$ benzylamine $(50 \mathrm{Ci} / \mathrm{mmol}$; ICN Biomedicals Inc., Irvine, $\mathrm{CA})$ as substrate (23). Measurements of platelet MAO activity were also carried out at the Academic Medical Center (Amsterdam, The Netherlands) using a fluorimetric method with kynuramine as substrate (24). 


\section{Cosmid C5}

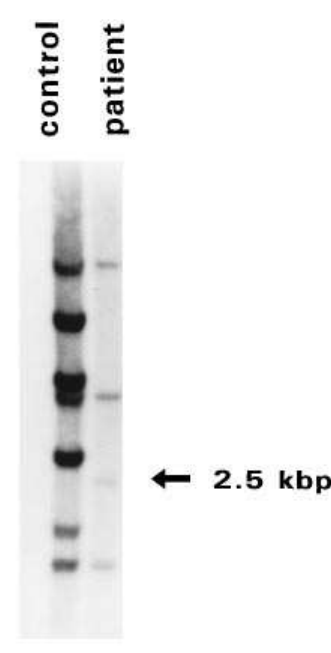

MAOB cDNA

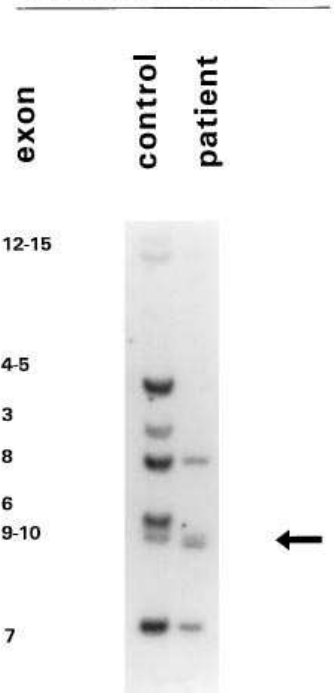

Figure 2. Fine mapping of the distal deletion breakpoint to within the MAO-B gene. (Left) Autoradiograph of a Southern blot containing EcoRI-digested DNA of a control and of one Norrie disease microdeletion patient after hybridization to cosmid clone C5 encompassing a large part of the MAO-B gene. A 2.5kbp junction fragment is indicated by an arrow. (Right) The same Southern blot hybridized to a MAO-B cDNA probe containing exons $3-15$. Bands corresponding to specific exons are indicated in the control. Exons 3-5 are lacking in the patient. Exon 6 is present, but the fragment size is altered, locating the breakpoint within intron 5 .
Platelet concentrations of 5-HT were measured at the NIH as described previously (5).

Plasma neurochemical assays. All assays of plasma catecholamines and their metabolites were carried out at the NIH. The catecholamines NE, EPI, and dopamine (DA); the deaminated catecholamine metabolites 3,4-dihydroxyphenylglycol (DHPG) and dihydroxyphenylacetic acid (DOPAC); and the catecholamine precursor dihydroxyphenylalanine (DOPA) were extracted from 1-ml samples of plasma using alumina and quantified by liquid chromatography with electrochemical detection (25). The metanephrines-normetanephrine and metanephrine, O-methylated amine metabolites of NE and EPI, respectively-were extracted from 2-ml samples of plasma using solidphase ion exchange columns and quantified by liquid chromatography with electrochemical detection (26). Timed collections of the catecholamine and metanephrine eluants leaving the electrochemical cell, followed by liquid scintillation spectroscopy, were used to determine plasma concentrations of ${ }^{3} \mathrm{H}$-labeled catecholamines and metanephrines. Concentrations of sulfate-conjugated metanephrines were determined after deconjugation by incubation with sulfatase (Sigma Chemical Co., St. Louis, MO). Liquid chromatography with electrochemical detection was also used for measurement of the deaminated NE metabolite, 3-methoxy-4-hydroxyphenylglycol (MHPG), after extraction from plasma into ethylacetate (27). Concentrations of the DA metabolite, homovanillic acid (HVA), and of the final deaminated end-product of NE and EPI metabolism, vanillylmandelic acid (VMA), were measured by gas chromatography-mass spectrometry after ethylacetate extraction and derivatization with pentafluoropropionic anhydride (28).

Urine neurochemical assays. Urine concentrations of catecholamines and their metabolites were determined at two centers: the $\mathrm{NIH}$ and the Academic Medical Center. These measurements were carried out using gas chromatography-mass spectroscopy at the NIH $(8,28)$ and liquid chromatography with fluorimetric detection at the Academic Medical Center (29, 30). Urine concentrations of 5-HT were also measured at the latter center using liquid chromatography with fluorometric detection. Urine concentrations of trace amines (phenylethylamine, $m$-tyramine, and $p$-tyramine) were assayed at St. Elizabeth's Hospital (Washington, DC) by gas chromatography-mass spectroscopy as described previously (31). Urine concentrations of catecholamines and their metabolites are reported as total concentrations (free and conjugated). Some of the urine data for the MAO$\mathrm{AB}-$ and MAO-A-deficient subjects have been reported elsewhere $(5,6,8,11)$.
Data analysis. The mean and SD of the plasma concentrations of catecholamines and metabolites in the 29 control subjects were calculated and served as the reference group for the tabulated data. Reference values for urine catecholamines and their metabolites were used from the original published methods. Differences between MAOdeficient subjects and control groups were tested by the Mann-Whitney U test. Within subject differences were determined using the Wilcoxon signed rank sum test. Differences were considered significant at $P<0.05$ (two tailed). All values in the text are presented as mean \pm SEM, unless indicated otherwise.

\section{Results}

Clinical characteristics of the $M A O-B$-deficient subjects. A general physical examination of the two brothers with the MAO-B deficiency, including global neurological examination, was unremarkable. They have normal blood pressure and heart rate. They are blind and have impaired hearing as part of Norrie disease. Psychomotor development is normal. Both subjects have completed university degrees.

Delineation of the X chromosome microdeletion. We have previously reported that the deletion in these two brothers with Norrie disease extends from within the Norrie disease gene on the proximal side to within the MAO-B gene (17). Precise mapping of the deletion breakpoint was achieved by hybridization of the patients DNA to 13 cosmids derived from a 300-kb contig that encompasses the MAO-B and Norrie disease genes. In this way, the size of the deletion was estimated to be $150 \mathrm{kbp}$. Moreover, when cosmid C5 was used as a hybridization probe on EcoRI-digested genomic DNA, four of the nine fragments were absent in the patient, and a putative deletion junction fragment of $2.5 \mathrm{kbp}$ appeared (Fig. 2, left). Cosmid C5 had been shown previously to cover most of the MAO-B gene (17).

Deletion of the $5^{\prime}$ end of the MAO-B gene was confirmed by hybridizing the same Southern blot to a cDNA probe containing exons 3-15 of the MAO-B gene. This experiment demonstrated deletion of exons 3-5 and an altered size of the fragment containing exon 6 (Fig. 2, right). Based on these results, the distal deletion breakpoint is located in intron 5 , between 


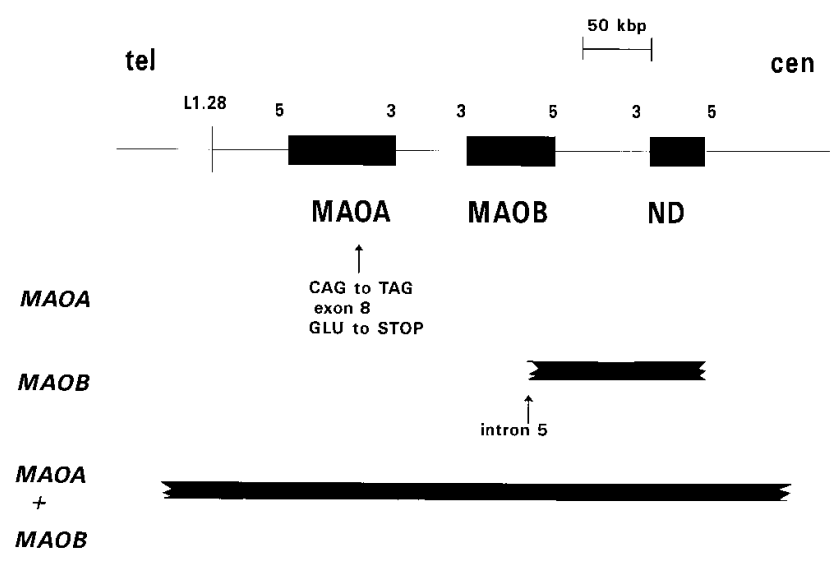

Figure 3. Overview of the MAO-A and MAO-B gene area in Xp11.3, based on Berger et al. (17) and Grimsby et al. (22). The location of the Norrie disease gene $(N D)$ is indicated. The molecular defect in three groups of patients is compared. MAOA, selective deficiency of MAO-A through a point mutation $\mathrm{C}$ to $\mathrm{T}$ in exon 8 of the MAO-A gene (Ref. 12, patients 3 and 4); $M A O B$, selective deficiency of MAO-B through a microdeletion encompassing the NDP gene and part of the MAO-B gene, leaving MAO-A intact (patients 1 and 2 in this study); $M A O A+M A O B$, combined deficiency of MAO-A and $\mathrm{MAO}-\mathrm{B}$ through a larger deletion encompassing all three genes, as well as the anonymous DNA marker L1.28 (DXS7).

exons 5 and 6 or possibly just within exon 6 (Fig. 3). Thus, the promoter, the transcription start site, and the first five exons that contain the DNA sequence encoding the first 159 amino acids of MAO-B are lacking in these patients.

Documentation of $M A O-B$ deficiency. Both assays of platelet MAO-B activity, carried out at separate centers using either benzylamine or kynuramine as substrates, indicated undetectable levels of MAO-B activity in the two subjects with deletion of the MAO-B gene (Table I). Platelet MAO-B activity was also undetectable in patients with combined MAO-AB deficiency, whereas it was normal in the subjects with selective
MAO-A deficiency. The activity of plasma amine oxidase was normal in all three subject groups (Table I).

Platelet 5-HT. The 5-HT content of platelets was within normal limits in the two subjects with MAO-B deficiency, whereas it was increased considerably in the groups with selective MAO-A and combined MAO-AB deficiencies (Table I).

Plasma concentrations of catecholamines and metabolites. Plasma concentrations of NE and EPI were within the normal range in all MAO-deficient subjects (Table II). Plasma concentrations of DA were also normal in the subjects with selective deficiencies of MAO-B or MAO-A, whereas they were higher in patients with the combined MAO-AB deficiency than in control subjects $(0.19 \pm 0.06$ vs $0.07 \pm 0.01 \mathrm{pmol} / \mathrm{ml} ; P<$ 0.05). Plasma concentrations of free and sulfate-conjugated normetanephrine and metanephrine, the O-methylated amine metabolites of NE and EPI, were normal in MAO-B-deficient subjects, whereas both groups of MAO-A- and MAO-ABdeficient subjects showed considerable elevations above normal in plasma concentrations of free and sulfate-conjugated normetanephrine and sulfate-conjugated metanephrine (Table II). In contrast, all MAO-deficient subjects, apart from one MAO-AB-deficient patient (number 6), showed normal or only slightly elevated plasma concentrations of free metanephrine. Thus, plasma concentrations of sulfate-conjugated metanephrine in MAO-A- and MAO-AB-deficient subjects were elevated above mean concentrations of normal subjects more than concentrations of free metanephrine $(4.5 \pm 0.53$ vs $2.1 \pm$ 0.7 -fold increase; $P<0.03$ ). Similarly, plasma concentrations of sulfate-conjugated normetanephrine showed larger elevations in MAO-A- and MAO-AB-deficient subjects than concentrations of free normetanephrine (13.2 \pm 3.0 vs $6.5 \pm 0.6$-fold increase; $P<0.02)$. Increases in plasma concentrations of free and sulfate-conjugated normetanephrine were larger $(P<$ 0.02) than those of metanephrine.

Plasma concentrations of DHPG, the deaminated metabolite of NE and EPI, were normal in MAO-B-deficient subjects, whereas they were reduced by $>95 \%$ in MAO-A subjects and undetectable in four of the five subjects with combined MAO-AB deficiency (Table II). A similar pattern

Table I. Platelet MAO Activity (in nmol/10 ${ }^{8}$ Platelets per $h$ ), with Benzylamine and with Kynuramine as Substrates, Platelet 5-HT (nmol/10 ${ }^{8}$ Platelets) and Plasma Amine Oxidase (nmol/ml per h)

\begin{tabular}{|c|c|c|c|c|c|}
\hline Subject No. & $\begin{array}{c}\text { MAO } \\
\text { deficiency }\end{array}$ & $\begin{array}{c}\text { MAO-B activity } \\
\text { platelets } \\
\text { (benzylamine) }\end{array}$ & $\begin{array}{c}\text { MAO-B activity } \\
\text { platelets } \\
\text { (kynuramine) }\end{array}$ & $\begin{array}{c}5 \text {-HT } \\
\text { platelets }\end{array}$ & $\begin{array}{c}\text { Amine oxidase } \\
\text { plasma }\end{array}$ \\
\hline 1 & $\mathrm{~B}$ & $<\mathbf{0 . 0 2}$ & $<\mathbf{1 . 0}$ & 0.30 & 14.7 \\
\hline 2 & B & $<\mathbf{0 . 0 2}$ & $<\mathbf{1 . 0}$ & 0.17 & 16.4 \\
\hline 3 & A & 11.21 & 25.5 & 1.73 & 22.4 \\
\hline 4 & A & 12.10 & 22.5 & 1.59 & 15.4 \\
\hline 5 & $\mathrm{AB}$ & $<\mathbf{0 . 0 2}$ & ne & ne & 25.9 \\
\hline 6 & $\mathrm{AB}$ & $<\mathbf{0 . 0 2}$ & ne & ne & 33.1 \\
\hline 7 & $\mathrm{AB}$ & $<\mathbf{0 . 0 2}$ & ne & ne & 38.3 \\
\hline 8 & $\mathrm{AB}$ & $<\mathbf{0 . 0 2}$ & ne & 2.06 & 32.1 \\
\hline 9 & $\mathrm{AB}$ & $<\mathbf{0 . 0 2}$ & ne & 2.51 & 15.7 \\
\hline \multirow[t]{3}{*}{ Control subjects } & Mean & 10.7 & 17.6 & 0.25 & 21.3 \\
\hline & SD & 1.6 & 5.0 & 0.03 & 2.8 \\
\hline & $n$ & 12 & 17 & 12 & 12 \\
\hline
\end{tabular}

Compounds with abnormal values are depicted in bold. ne, not examined. 
Table II. Plasma Concentrations (nmol/liter) of Catecholamines and Metabolites in MAO-deficient Subjects and in Control Subjects

\begin{tabular}{|c|c|c|c|c|c|c|c|c|c|c|c|c|c|}
\hline \multirow[b]{2}{*}{ Subject } & \multirow[b]{2}{*}{ Deficiency } & \multicolumn{3}{|c|}{ Catecholamines } & \multicolumn{4}{|c|}{ O-methylated amine metabolites } & \multicolumn{5}{|c|}{ Deaminated metabolites } \\
\hline & & $\mathrm{NE}$ & EPI & DA & NMN & $\mathrm{NMN}-\mathrm{SO}_{4}$ & $\mathrm{MN}$ & $\mathrm{MN}-\mathrm{SO}_{4}$ & DHPG & MHPG & VMA & DOPAC & HVA \\
\hline 1 & $\mathrm{~B}$ & 2.37 & 0.10 & 0.14 & 0.32 & 11.7 & 0.16 & 5.1 & 4.91 & 11.5 & 29.7 & 9.8 & 49.8 \\
\hline 2 & B & 3.14 & 0.35 & 0.08 & 0.53 & 14.5 & 0.26 & 5.9 & 5.17 & 15.7 & 41.2 & 5.2 & 56.0 \\
\hline 3 & A & 0.89 & 0.10 & 0.02 & 1.19 & 39.9 & 0.09 & 10.6 & 0.51 & 1.1 & 3.5 & 4.0 & 20.9 \\
\hline 4 & A & 2.09 & 0.04 & 0.03 & 2.27 & 73.3 & 0.27 & 19.1 & 0.38 & 2.1 & 7.7 & 3.7 & 24.3 \\
\hline 5 & $\mathrm{AB}$ & 4.10 & 0.32 & 0.03 & 1.78 & 106.0 & 0.25 & 14.1 & $<\mathbf{0 . 0 3}$ & 0.2 & 2.2 & 11.4 & 46.9 \\
\hline 6 & $\mathrm{AB}$ & 2.66 & 0.17 & 0.15 & 1.97 & 86.1 & 0.95 & 16.4 & $<\mathbf{0 . 0 3}$ & 0.4 & 0.7 & 3.0 & 17.5 \\
\hline 7 & $\mathrm{AB}$ & 2.16 & 0.10 & 0.27 & 1.46 & 92.6 & 0.20 & 15.8 & $<\mathbf{0 . 0 3}$ & 0.2 & 1.1 & 1.4 & 4.9 \\
\hline 8 & $\mathrm{AB}$ & 2.91 & 0.11 & 0.37 & 2.29 & 234.0 & 0.23 & 9.4 & 0.12 & 0.3 & 2.2 & 0.5 & 12.1 \\
\hline 9 & $\mathrm{AB}$ & 1.94 & 0.04 & 0.13 & 1.37 & 85.6 & 0.21 & 7.7 & $<\mathbf{0 . 0 3}$ & 0.1 & 0.6 & 0.3 & 2.3 \\
\hline \multirow{3}{*}{$\begin{array}{l}\text { Control } \\
\text { subjects }\end{array}$} & Mean & 1.35 & 0.14 & 0.08 & 0.25 & 7.5 & 0.16 & 3.1 & 5.54 & 14.3 & 31.1 & 9.1 & 51.9 \\
\hline & $\mathrm{SD}$ & 0.59 & 0.09 & 0.04 & 0.11 & 5.0 & 0.06 & 1.2 & 1.37 & 2.1 & 2.5 & 5.6 & 9.0 \\
\hline & $n$ & 29 & 27 & 11 & 29 & 27 & 27 & 26 & 29 & 17 & 10 & 21 & 11 \\
\hline
\end{tabular}

Compounds with abnormal levels are depicted in bold. $N M N$, normetanephrine; $N M N$ - $S O_{4}$, sulfate-conjugated normetanephrine; $M N$, metanephrine; $\mathrm{MN}_{-} \mathrm{SO}_{4}$, sulfate-conjugated metanephrine.

was observed for MHPG and VMA, the deaminated and O-methylated metabolites of NE and EPI; these metabolites were normal in MAO-B-deficient subjects, whereas they were reduced by $75-92 \%$ in the two MAO-A-deficient subjects and reduced further to $>93 \%$ in the subjects with the combined MAO-AB deficiency.

Since plasma concentrations of DHPG and sulfate-conjugated normetanephrine showed the largest reciprocal changes in MAO-AB-deficient subjects, the ratios of these metabolites were compared with those in normal subjects and patients receiving the MAO inhibitor, deprenyl (Fig. 4). Ratios of plasma concentrations of sulfate-conjugated normetanephrine to DHPG were within the normal range in the MAO-B-deficient subjects, whereas these ratios were elevated by $\sim 100$-fold in the

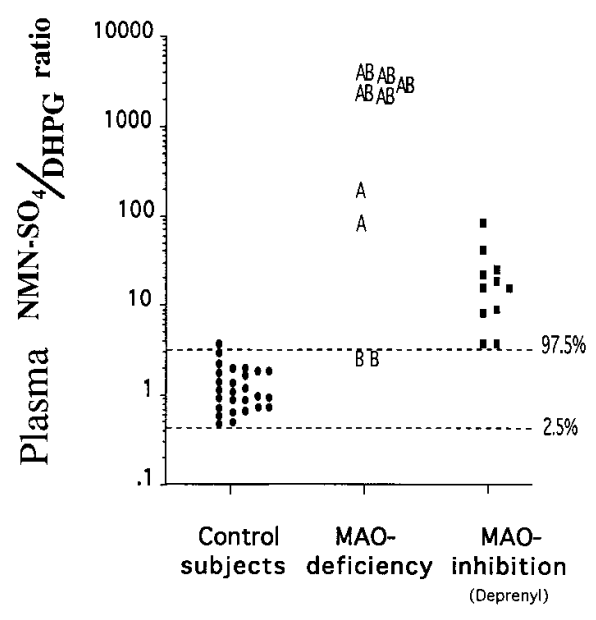

Figure 4. The plasma normetanephrine $(N M N)-\mathrm{SO}_{4} / \mathrm{DHPG}$ ratio in the patients with MAO deficiency, in control subjects, and in the subjects treated with the MAO-B inhibitor, deprenyl. $A B$, combined MAO-AB deficiency; $A$, MAO-A deficiency; $B$, MAO-B deficiency. The dashed lines represent the upper and lower reference values as determined from the 2.5 and 97.5 percentile values of the control subjects.
MAO-A-deficient subjects and by more than several thousand-fold in all five subjects with the combined MAO-AB deficiency. Similarly, in the subjects who received deprenyl, a $250 \%$ increase in conjugated normetanephrine and a $60 \%$ decrease in plasma concentrations of DHPG resulted in an increase in the ratio of these two metabolites.

The deaminated metabolites of DA, DOPAC, and HVA showed a less marked and more variable trend than that observed for the deaminated metabolites of NE and EPI (Table II). Again, plasma concentrations of these metabolites were normal in the two MAO-B-deficient subjects but reduced by $53-60 \%$ in the MAO-A-deficient subjects. Plasma DOPAC and HVA tended to be reduced more in the combined MAOAB-deficient subjects than in the two MAO-A-deficient subjects, but there was considerable variability. In particular, one subject with combined MAO-AB deficiency showed normal concentrations of both DOPAC and HVA.

Plasma levels of DOPA, the catecholamine precursor, were normal in MAO-B-deficient subjects (7.65 and $7.54 \mathrm{nmol} / \mathrm{li}-$ ter), but significantly lower in subjects with selective MAO-A and combined MAO-AB deficiencies than in control subjects (5.64 \pm 0.44 vs $8.55 \pm 0.40 \mathrm{nmol} /$ liter, respectively; $P<0.01$ ).

Urinary excretion of catecholamines, catecholamine metabolites, 5-HT, and trace amines. The only abnormal neurochemical finding in the MAO-B-deficient subjects was an increase in urinary phenylethylamine levels (Table III). Urinary excretion of the other trace amines, meta- and paratyramine, was normal, as was that of the catecholamines, their O-methylated and deaminated metabolites, and 5-HT.

In contrast to the MAO-B-deficient subjects, the two subjects with a selective deficiency of MAO-A showed increased excretion of 5-HT, normetanephrine, and the O-methylated amine metabolite of DA, 3-methoxytyramine (11). These subjects also showed decreased or low-normal urinary excretion of the deaminated metabolites, MHPG, VMA, and HVA. Excretion of all trace amines was within normal limits.

In subjects with the combined MAO-AB deficiency, the urinary excretion of catecholamines and their metabolites 
Table III. Urinary Excretion of Catecholamines, Catecholamine Metabolites, 5-HT and Trace Amines in MAO-deficient Subjects and in Control Subjects (nmol/mmol Creatinine)

\begin{tabular}{|c|c|c|c|c|c|c|c|c|c|c|c|c|c|c|}
\hline \multirow[b]{2}{*}{ Subject No. } & \multirow{2}{*}{$\begin{array}{c}\text { MAO } \\
\text { deficiency }\end{array}$} & \multicolumn{3}{|c|}{ Catecholamines } & \multicolumn{3}{|c|}{ O-methylated metabolites } & \multicolumn{3}{|c|}{ Deaminated metabolites } & \multirow[b]{2}{*}{$5-\mathrm{HT}$} & \multicolumn{3}{|c|}{ Trace amines } \\
\hline & & $\mathrm{NE}$ & EPI & DA & NMN & MN & 3-MT & MHPG & VMA & HVA & & PE & m-Tyr & p-Tyr \\
\hline 1 & B & 165 & ne & 558 & 203 & 72 & 76 & 900 & 1,300 & 1,700 & 50 & 76 & 35 & 271 \\
\hline 2 & B & 128 & 26 & 536 & 131 & 44 & 126 & 700 & 1,900 & 1,500 & 57 & 207 & 48 & 320 \\
\hline 3 & A & 62 & 8 & 730 & 392 & 56 & 217 & 100 & 200 & 400 & 124 & 4.0 & 18 & 499 \\
\hline 4 & A & 142 & 11 & 803 & 666 & 82 & 318 & 200 & 400 & 500 & 233 & 8.0 & 33 & 201 \\
\hline 9 & $\mathrm{AB}$ & 106 & 189 & 905 & 1,524 & 162 & 1,537 & 61 & $<\mathbf{2 0 0}$ & 700 & 256 & 149 & 1,344 & $\mathbf{3 , 8 4 0}$ \\
\hline $\begin{array}{c}\text { Controls of } \\
\text { AMC }\end{array}$ & range & $27-146$ & $1.6-21$ & $147-951$ & $34-182$ & $10-90$ & $37-118$ & $100-2,100$ & $1,000-5,000$ & $500-1,700$ & $11-68$ & $(\mathrm{Se}$ & e below & \\
\hline 5 & $\mathrm{AB}$ & 300 & ne & 2,666 & 2,537 & 166 & 1,766 & 86 & 251 & 2,005 & ne & 1,012 & 332 & 2,001 \\
\hline 6 & $\mathrm{AB}$ & 461 & ne & 1,957 & 2,390 & 224 & 1,711 & 80 & 200 & 5,103 & ne & 1,816 & 425 & 2,492 \\
\hline 7 & $\mathrm{AB}$ & 441 & ne & 1,920 & 2,908 & 247 & 2,910 & 111 & 508 & 2,266 & ne & 9,423 & 667 & 8,499 \\
\hline 8 & $\mathrm{AB}$ & 388 & ne & 1,041 & 1,840 & 178 & 947 & 55 & 223 & 360 & ne & 4,159 & 1,030 & 5,295 \\
\hline $\begin{array}{l}\text { Controls of } \\
\text { NIH }\end{array}$ & $\begin{array}{c}\text { Mean } \\
\text { SD }\end{array}$ & $\begin{array}{l}180 \\
127\end{array}$ & & $\begin{array}{l}516 \\
162\end{array}$ & $\begin{array}{r}240 \\
74\end{array}$ & $\begin{array}{l}63 \\
34\end{array}$ & $\begin{array}{l}40 \\
10\end{array}$ & $\begin{array}{l}805 \\
129\end{array}$ & $\begin{array}{r}1,849 \\
519\end{array}$ & $\begin{array}{l}3,564 \\
1,440\end{array}$ & - & $\begin{array}{l}6.8 \\
4.1\end{array}$ & $\begin{array}{l}43 \\
12\end{array}$ & $\begin{array}{r}361 \\
71\end{array}$ \\
\hline
\end{tabular}

Compounds with abnormal values are depicted in bold. Academic Medical Center Amsterdam control values for catecholamines, catecholamine metabolites, and 5-HT apply to subjects 1-4 and 9. National Institutes of Health control values for catecholamines and catecholamine metabolites apply to subjects 5-8. All trace amines were assayed at St. Elizabeth's Hospital, Washington, DC. $N M N$, normetanephrine; $M N$, metanephrine; 3-MT, 3-methoxytyramine; $P E$, phenylethylamine; $m$-Tyr, m-tyramine; $p$-Tyr, p-tyramine; $A M C$, Academic Medical Center Amsterdam; NIH, National Institutes of Health; ne, not examined.

showed similar qualitative alterations as in subjects with the selective MAO-A deficiency but tended to be more variably disturbed (Table III). In particular, excretion of HVA showed highly variable changes compared with other subject groups. Similar to the situation in plasma, urinary levels of DA were also increased in patients with combined MAO-AB deficiencies but not in those with selective deficiencies of either isoenzyme.

Excretion of all trace amines was profoundly increased in patients with combined MAO-AB deficiency. In particular, excretion of phenylethylamine was increased substantially more in combined MAO-AB-deficient subjects than in selective MAO-B-deficient subjects. Furthermore, whereas excretion of the tyramine trace amines was normal in subjects with either selective deficiency of MAO-A or MAO-B, it was increased considerably in subjects with the combined MAO-AB deficiency.

Forearm spillover of $N E$ and normetanephrine in MAO-A deficiency. Use of the tracer-labeled NE and EPI isotope dilution technique indicated an increase in forearm spillover into plasma of normetanephrine in the two MAO-A-deficient subjects (Table IV). Forearm spillovers of normetanephrine from both circulating and locally released NE were increased. Forearm spillover of NE was also increased in one of these subjects.

Tyramine sensitivity test. In one of the two MAO-A-deficient subjects, systolic blood pressure was increased to the 30$\mathrm{mmHg}$ end-point by an intravenous dose of tyramine of only $0.11 \mathrm{mg} / \mathrm{min}$; this represents a dose less than one-tenth of that required in a control group of 11 patients with depression (19), and comparable to that in patients treated with selective MAO-A and nonselective MAO inhibitors (32), but about fourfold more than the end-point dose required in a juvenile patient with combined MAO-AB deficiency (5). In the other MAO-A-deficient subject, the tyramine infusion had to be stopped prematurely at an infusion rate of $0.03 \mathrm{mg} / \mathrm{min}$ because of persisting oppressing pain in his chest. Systolic blood pressure had increased by $5 \mathrm{mmHg}$ at this infusion rate.

\section{Discussion}

This is the first description of patients with a selective loss of MAO-B activity due to a disruption of the MAO-B gene on the $\mathrm{X}$ chromosome. This is also the first comprehensive report comparing neurochemical and clinical phenotypes in patients with selective deficiencies of MAO-A and MAO-B and combined deficiencies of MAO-AB. The results demonstrate that the three groups of patients each display characteristic neuro-

Table IV. Forearm Spillover and Removal Rates of NE and Normetanephrine (NMN) in the Two Subjects with MAO-A Deficiency and in Control Subjects $(n=10)$

\begin{tabular}{lccccc}
\hline & & & \multicolumn{3}{c}{ Forearm NMN spillover } \\
\cline { 3 - 6 } Subject & NE spillover & NE removal & Circulating NE & Local NE & Total \\
\hline 3 & 262 & 185 & 6.7 & $\mathbf{1 5 3}$ & $\mathbf{1 6 0}$ \\
4 & $\mathbf{6 4 3}$ & 406 & $\mathbf{2 5 . 7}$ & $\mathbf{2 6 8}$ & $\mathbf{2 9 4}$ \\
Control subjects & $388 \pm 199$ & $245 \pm 155$ & $3.6 \pm 5.6$ & $51 \pm 52$ & $54 \pm 52$
\end{tabular}

Forearm normetanephrine spillovers represent those derived from forearm removal of circulating $\mathrm{NE}$, locally released $\mathrm{NE}$, and the total of both. Values represent mean \pm SD ( $\mathrm{pmol} / \mathrm{min})$. Abnormal values are depicted in bold. 
chemical profiles that may contribute to the different clinical phenotypes of each group.

Genotype and clinical phenotype of $M A O-B$ deficiency patients. In addition to a partial deletion of the MAO-B gene, the gene for Norrie disease is also involved in the syndrome affecting these two brothers. In fact, these patients were originally described as part of a previous study of Norrie disease that resulted in the cloning of the Norrie disease gene (17). Although these subjects show the characteristic features of Norrie disease, namely early onset blindness and progressive hearing loss, they show no signs of physical or mental impairment. In particular, no signs of mental retardation or behavioral disturbances have been noted as are observed in patients with classical Norrie disease combined with MAO-AB deficiency (5-8) or in subjects with selective MAO-A deficiency $(11,12)$. Only a part of the MAO-B gene is deleted, but since this includes the DNA sequence encoding the first 159 amino acids of MAO-B, this is incompatible with any functional MAO-B activity. A complete and selective lack of MAO-B is documented by the undetectable levels of platelet MAO-B activity, determined by two different assay techniques, and is also reflected in the increased urinary phenylethylamine levels that are characteristically found with reduced MAO-B activity (33).

Metabolic roles of MAO isoenzymes. Comparison of the selective deficiencies of MAO-A and MAO-B and combined deficiency of MAO-AB provides a unique insight into the metabolic roles of the MAO isoenzymes in humans. This insight is not only important for interpreting the clinical consequences of MAO deficiency states, but also for understanding the basis of therapeutic interventions involving selective inhibition of MAO-A or MAO-B. Up until now, studies of the metabolic roles of MAO-A and MAO-B have been confined largely to pharmacological studies in animals or isolated tissues. Such studies are limited by a lack of completely selective MAO inhibitors as well as by difficulties in extrapolating the function of MAO from isolated tissues or animals to humans (3).

The severely impaired deamination of catecholamines in subjects with combined MAO-AB deficiency is demonstrated by the $>93 \%$ reductions in plasma concentrations of the deaminated catecholamine metabolites, DHPG, MHPG, and VMA. Additionally, the markedly elevated plasma concentrations of O-methylated amine metabolites-free and sulfateconjugated normetanephrine and sulfate-conjugated metanephrine-reflect impaired deamination of these metabolites as well as increased availability of the parent amines for O-methylation (14). Slightly less severe changes in patients with selective MAO-A deficiency and complete lack of similar directional changes in MAO-B-deficient subjects indicate that MAO-A is considerably more important than MAO-B for metabolism of NE, EPI, and their O-methylated amine metabolites in vivo. These findings agree with those in rats (14), where pharmacological inhibition of MAO-B failed to affect either the deaminated or O-methylated amine metabolites of $\mathrm{NE}$ and EPI, whereas inhibition of MAO-A resulted in similar directional changes to those observed here.

Larger increases in plasma concentrations and urinary excretion of normetanephrine than of metanephrine are also in agreement with results in rats or humans administered MAO inhibitors, a difference attributed to little deamination of EPI and metanephrine in the compartment where metanephrine is formed $(14,15)$. The larger increases in plasma concentrations of sulfate-conjugated than free metanephrines can be explained similarly by compartmental differences in sites of formation. Over $90 \%$ of free metanephrine and a substantial, but lower, portion of free normetanephrine are formed within the adrenals $(16,18)$. In contrast, the conjugated metanephrines are not formed within the adrenals, but are formed elsewhere in the body where deamination is a more prominent pathway than in the adrenals.

As previously described (12), cultured skin fibroblasts of the two subjects with the selective MAO-A deficiency had no MAO-A activity. The present study extends the observation of MAO-A deficiency to the organ level and documents impaired extraneuronal deamination of NE and normetanephrine as indicated by the increased forearm spillover of normetanephrine from locally released and circulating NE.

According to a study in isolated human brain tissue, DA was proposed as a selective substrate for MAO-B (34). However, studies of substrate specificity of the two forms of MAO in isolated tissues do not always reflect reliably the situation in vivo, and it has now been established that, under physiological conditions, DA is also a substrate for MAO-A (3). The decreases in plasma DOPAC and HVA, decreases in urinary excretion of HVA, and increases in urinary excretion of the O-methylated DA metabolite, 3-methoxytyramine, in MAOA-deficient subjects support an important role for MAO-A in the metabolism of DA. This finding and the complete lack of any similar directional changes in the MAO-B-deficient subjects are in agreement with the results of individual blockade of the two isoenzymes in rats (14) and raise questions about whether MAO-B has any physiological role in the normal in vivo metabolism of DA.

The substantial decreases in deaminated catecholamine metabolites and increases in O-methylated amine metabolites in MAO-A-deficient but not MAO-B-deficient subjects indicate that the two isoenzymes do not have equal complementary capacities for the deamination of catecholamines when one of the two isoenzymes is lacking. Nevertheless, the somewhat exaggerated neurochemical changes in patients with combined MAO-AB deficiency compared with selective MAO-A deficiency indicate that MAO-B plays a minor auxiliary role in the metabolism of catecholamines when the activity of MAO-A is impaired. This conclusion is further supported by the elevated plasma concentrations and urinary excretion of DA in MAO-AB-deficient subjects, but not in other groups. In rats, combined inhibition of the two MAO isoenzymes reduces the deamination of intravenously infused NE more than inhibition of MAO-A alone, whereas inhibition of MAO-B alone has no effect (14). The results indicate that, under normal conditions, MAO-B does not have a major role in the deamination of catecholamines. Normal urinary and platelet levels of 5-HT in MAO-B-deficient subjects and substantial increases in selective MAO-A- and combined MAO-AB-deficient subjects also suggest that MAO-B is of less importance than MAO-A for the in vivo metabolism of 5-HT.

An important role for MAO-B is, however, evident for deamination of the trace amines, phenylethylamine and paraand metatyramine. Increased urinary phenylethylamine in MAO-B-deficient subjects represents the only neurochemical abnormality we could detect in these subjects. This increase contrasted with normal urinary phenylethylamine levels in MAO-A-deficient subjects, findings consistent with the reported preferential deamination of phenylethylamine by MAO-B (1, 
33). The larger increases in phenylethylamine in the individuals with combined MAO-AB deficiencies relative to those with MAO-B deficiency alone indicate that MAO-A can partially substitute for MAO-B in degrading this trace amine in vivo. In contrast to phenylethylamine, urinary tyramine excretion was not increased in subjects with selective deficiency of MAO-B or MAO-A but was profoundly increased in MAO$\mathrm{AB}$-deficient subjects. This confirms that tyramine is a substrate for both MAO-A and MAO-B (35) and represents one situation in which one isoenzyme is able to compensate fully for the absence of the other. Increased pressor sensitivity to tyramine in the subjects with selective deficiency of MAO-A observed here, in addition to that previously described in MAO-AB-deficient subjects described elsewhere (5), can be explained by dependence of these sensitivity changes on the capacity to deaminate both tyramine and the NE released from vesicles by tyramine (19). Predominance of MAO-A within sympathetic neurons (1), where tyramine exerts its pressor actions, is another factor.

Normal plasma concentrations of DOPA in MAO-B-deficient subjects and lowered concentrations in subjects with deficiency of MAO-A, alone or in combination with MAO-B, reflect the important role of MAO-A in the metabolic degradation of NE within the neuronal cytoplasm (1). As previously demonstrated, increased concentrations of $\mathrm{NE}$ in the neuronal cytoplasm during inhibition of intraneuronal MAO results in feedback inhibition of tyrosine hydroxylase and decreased formation and plasma concentrations of the immediate product of the enzyme, DOPA $(15,25,36)$.

The presence of detectable amounts of deaminated metabolites of catecholamines, particularly those of DA, in patients with neither MAO-A nor MAO-B activities indicates that these amines or their O-methylated derivatives are substrates, albeit weak substrates, for other enzymes that degrade amines. Plasma amine oxidase represents one such enzyme that in humans can deaminate both DA and its O-methylated metabolite, 3-methoxytyramine $(37,38)$, and which is present at normal levels in patients with a lack of MAO isoenzymes.

$M A O$ deficiency and behavior. MAO is a pivotal determinant in the metabolism of amines that affect behavior and mood; pharmacological inhibition of MAO in animals and humans has profound effects on neurophysiological functioning $(39,40)$. Consequently, it has been assumed that genetically determined variations of MAO activity might be associated with behavioral and mood disorders. Numerous cross-sectional studies have established an association between low platelet MAO-B activity and pathological behavior (41-43); a causal relation, however, has never been established. A lack of correlation in MAO-B activity between platelets and brain has been suggested (44). However, the amino acid sequences are identical (45), and the biochemical and pharmacological characteristics of the isoenzyme in the two tissues are also similar (35), excluding a genetically encoded difference in the structure of the MAO-B isoenzyme between these tissues.

The present study shows that lack of MAO-B is associated with apparently normal behavior, whereas a lack of MAO-A is not $(11,12)$. In addition, the metabolic data in the MAO-Bdeficient subjects indicate that an impairment in the breakdown of phenylethylamine is not related to abnormal behavior. Normal phenylethylamine excretion in the selective MAO-A-deficient subjects further suggests that this trace amine is unlikely to be involved in the behavioral disorders that characterize these patients. These data do not, however, exclude a possible neuromodulatory function of phenylethylamine in central nervous system catecholamine systems (46).

Despite the predominance of MAO-B over MAO-A in the human brain (3), the prominent disturbances in behavior in MAO-A-deficient subjects and the normal behavior in MAO$\mathrm{B}$-deficient subjects indicate that MAO-A plays a more vital role than MAO-B in the normal neurophysiological development and function. This conclusion is consistent with the considerably more important role of MAO-A compared with MAO-B in the metabolism of catecholamines and 5-HT revealed by the present study. However, whether any of the profound neurochemical derangements observed in MAO-Adeficient subjects reflect the physiological basis of the behavioral disorder in these subjects remains to be established.

A relationship between the observed neurochemical and behavioral abnormalities in MAO-A-deficient subjects is strongly supported by a recent study in transgenic mice with a deletion in the MAO-A gene (47). The severe behavioral disturbances in these animals were associated with markedly elevated brain 5-HT concentrations and could be reversed by postnatal administration of a 5-HT synthesis inhibitor. A causal relationship between altered 5-HT metabolism and disturbed behavior was suggested to be mediated by discrete cortical alterations induced by chronically elevated 5-HT levels. Further support for involvement of altered 5-HT metabolism is provided by another recent study, in which behavioral disturbances in rats administered MAO inhibitors, before and after birth, were also associated with structural changes in brain serotoninergic systems (48). We have previously argued that the possible disruptive effect of raised 5-HT levels on the developing brain may help explain why impulsive aggressive behavior has been consistently associated with low levels of 5-HT or its primary metabolite, 5-hydroxyindolacetic acid, whereas 5-HT levels are elevated in subjects with selective MAO-A deficiency who show similar behavior (49).

The patients with combined deletions of the MAO-A and MAO-B genes also have deletions of the Norrie disease gene. However, the severe neurological symptoms and mental retardation in these patients are not universal characteristics of patients with Norrie disease $(8,16)$. This is illustrated by the present patients with deletions of the MAO-B and Norrie disease genes, both of whom had completed university degrees and in whom the only symptoms were the early onset blindness and impaired hearing. Despite the essentially normal neurochemical profile in MAO-B-deficient patients, those with combined MAO-AB deficiency had somewhat more severe neurochemical changes than MAO-A-deficient patients, suggesting that MAO-B may compensate, albeit to a limited extent, for lack of MAO-A. Thus, it is possible that the much more severe mental retardation and other neurological abnormalities observed in patients with combined MAO-AB (compared with selective MAO-A deficiency) are due to additional deletion of the MAO-B gene. However, since the patients with combined MAO-AB deficiency have deletions of various sizes that possibly involve other genes on the $\mathrm{X}$ chromosome, other explanations should also be considered.

Neurochemical phenotyping may provide a sensitive tool for detection of the MAO-A deficiency state. In contrast to laborious methods of direct DNA analysis and measurements of fibroblast MAO-A activities, measurement of plasma catecholamine metabolites provides a relatively simple screening 
procedure. In particular, use of the ratio of plasma concentrations of sulfate-conjugated normetanephrine to plasma DHPG, which are the metabolites that show the most dramatic changes, provides a sensitive method for the detection of subjects suspected of having reduced activity of MAO-A.

In conclusion, selective deficiency of MAO-B does not lead to a specific clinical phenotype and, in particular, is not associated with clinically apparent disturbances in behavior such as are observed in deficiencies of MAO-A. The absence in MAO-B-deficient patients of the severe neurochemical alterations observed in patients with deficiencies of MAO-A or $\mathrm{MAO}-\mathrm{AB}$ indicates that MAO-A is considerably more important than MAO-B for metabolism of most biogenic amines. These functional differences in the two isoenzymes of MAO are likely to contribute to the different clinical phenotypes observed in patients with deficiencies of either or both isoenzymes and may help in understanding the basis for therapeutic interventions involving specific inhibition of one or the other isoenzymes.

\section{Acknowledgments}

We gratefully acknowledge the contributions of our many co-authors on previously published papers who helped characterize the genetic, neurochemical, and behavioral features of the subjects reviewed in this paper. We also gratefully acknowledge the technical assistance of Jacques J. Willemsen, Douglas Hooper, Teresa Tolliver, and Jerry Oliver and the help of Marie-Cecile Jacobs in the studies of catecholamine kinetics.

\section{References}

1. Youdim, M.B.H., J.P.M. Finberg, and K.F. Tipton. 1988. Monoamine oxidase. In Catecholamines I. Handbook of Experimental Pharmacology. U. Trendelenburg and N. Weiner, editors. Springer Verlag, New York. 119-192.

2. Weyler, W., Y.-P.P. Hsu, and X.O. Breakefield. 1990. Biochemistry and genetics of monoamine-oxidase. Pharmacol. \& Ther. 47:391-417.

3. Berry, M.D., A.V. Juorio, and I.A. Paterson. 1994. The functional role of monoamine oxidases A and B in the mammalian central nervous system. Progr. Neurobiol. (Oxf.). 42:375-391.

4. Kochersperger, L.M., E.L. Parker, M. Siciliano, G.J. Darlington, and R.M. Denney. 1986. Assignment of genes for human monoamine oxidases A and B to the X-chromosome. J. Neurosci. 16:601-616.

5. Collins, F.A., D.L. Murphy, A.L. Reiss, K.B. Sims, J.G. Lewis, L. Freund, F. Karoum, D. Zhu, I.H. Maumenee, and S.E. Antonarakis. 1992. Clinical, biochemical and neuropsychiatric evaluation of a patient with a contiguous gene syndrome due to a microdeletion Xp11.3 including the Norrie disease locus and monoamine oxidase (MAOA and MAOB) genes. Am. J. Med. Genet. 42:127134.

6. Sims, K.B., A. de la Chapelle, R. Norio, E.-M. Sankila, Y.-P.P. Hsu, W.B. Rinehart, T.J. Corey, L. Ozelius, J.F. Powell, G. Bruns, et al. 1989. Monoamine oxidase deficiency in males with an $\mathrm{X}$ chromosome deletion. Neuron. 2:10691076.

7. Bleeker-Wagemakers, E.M., I. Zweije-Hofman, and A. Gal. 1988. Norrie disease as part of a complex syndrome explained by a submicroscopic deletion of the X chromosome. Ophthalmic. Paediatr. Genet. 9:137-142.

8. Murphy, D.L., K.B. Sims, F. Karoum, A. de la Chapelle, R. Norio, E.-M. Sankila, and X.O. Breakefield. 1990. Marked amine and amine metabolite changes in Norrie disease patients with an X-chromosomal deletion affecting monoamine oxidase. J. Neurochem. 54:242-247.

9. Donnai, D., R.C. Mountford, and A.P. Read. 1988. Norrie disease resulting from a gene deletion: clinical features and DNA studies. J. Med. Genet. 25: 73-78.

10. Murphy, D.L., K.B. Sims, F. Karoum, N.A. Garrick, A. de la Chapelle, E.M. Sankila, R. Norio, and X.O. Breakefield. 1991. Plasma amine oxidase activities in Norrie disease patients with an X-chromosomal deletion affecting monoamine oxidase. J. Neural Transm. 83:1-12.

11. Brunner, H.G., M.R. Nelen, P. van Zandvoort, N.G.G.M. Abeling, A.H. van Gennip, E.C. Wolters, M.A. Kuiper, H.H. Ropers, and B.A. van Oost. 1993. X-linked borderline mental retardation with prominent behavioral disturbance: phenotype, genetic localization, and evidence for disturbed monoamine metabolism. Am. J. Hum. Genet. 52:1032-1039.
12. Brunner, H.G., M. Nelen, X.O. Breakefield, H.H. Ropers, and B.A. van Oost. 1993. Abnormal behavior associated with a point mutation in the structural gene for monoamine oxidase A. Science (Wash. DC). 262:578-580.

13. Kopin, I.J. 1985. Catecholamine metabolism: basic aspects and clinical significance. Pharmacol. Rev. 37:333-364.

14. Eisenhofer, G., and J.P.M. Finberg. 1994. Different metabolism of norepinephrine and epinephrine by catechol-O-methyltransferase and monoamine oxidase in rats. J. Pharmacol. Exp. Ther. 268:1242-1251.

15. Eisenhofer, G., J.W.M. Lenders, J. Harvey-White, M. Ernst, A. Zametkin, D.L. Murphy, and I.J. Kopin. 1996. Differential inhibition of neuronal and extraneuronal monoamine oxidase. Neuropsychopharmacology. In press.

16. Eisenhofer, G., P. Friberg, K. Pacak, D.S. Goldstein, D.L. Murphy, C. Tsigos, A.A. Quyyumi, H.G. Brunner, and J.W.M. Lenders. 1995. Plasma metadrenalines: do they provide useful information about sympatho-adrenal function and catecholamine metabolism? Clin. Sci. (Lond.). 88:533-542.

17. Berger, W., A. Meindl, T.J.R. van de Pol, F.P.M. Cremers, H.H. Ropers, C. Döerner, A. Monaco, A.A.B. Bergen, R. Lebo, M. Warburg, et al. 1992. Isolation of a candidate gene for Norrie disease by positional cloning. Nat. Genet. 1:199-203.

18. Eisenhofer, G., B. Rundquist, A. Åneman, P. Friberg, N. Dakak, I.J. Kopin, M.C. Jacobs, and J.W.M. Lenders. 1995. Regional release and removal of catecholamines and extraneuronal metabolism to metanephrines. J. Clin. Endocrinol. \& Metab. 80:3009-3017.

19. Sunderland, T., E.A. Mueller, R.M. Cohen, D.C. Jimerson, D. Pickar, and D.L. Murphy. 1985. Tyramine pressor sensitivity changes during deprenyl treatment. Psychopharmacology. 86:432-437.

20. Miller, S.A., D.D. Dykes, and H.F. Polesky. 1988. A simple salting out procedure for extracting DNA from human nucleated cells. Nucleic Acids Res. 16:1215.

21. Sims, K.B., R.V. Lebo, G. Benso, C. Shalish, D. Schuback, Z.Y. Chen, G. Bruns, I.W. Craig, M.S. Golbus, and X.O. Breakefield. 1992. The Norrie disease gene maps to a $150 \mathrm{~kb}$ region on chromosome Xp11.3. Hum. Mol. Genet. $1: 83-89$.

22. Grimsby, J., K. Chen, L.-J. Wang, N.C. Lan, and J.C. Shih. 1991. Human monoamine oxidase $\mathrm{A}$ and $\mathrm{B}$ genes exhibit identical exon-intron organization. Proc. Natl. Acad. Sci. USA. 88:3637-3641.

23. Murphy, D.L., C. Wright, M. Buchsbaum, J. Costa, A. Nichols, and R. Wyatt. 1976. Platelet and plasma amine activities in 650 normals: sex and age differences and stability over time. Biochem. Med. 16:254-265.

24. van Kempen, G.M.J., J.L. van Brussel, and E.J.M. Penning. 1985. Assay of platelet monoamine oxidase in whole blood. Clin. Chim. Acta. 153:197-202.

25. Eisenhofer, G., D.S. Goldstein, R. Stull, H.R. Keiser, T. Sunderland, D.L. Murphy, and I.J. Kopin. 1986. Simultaneous liquid chromatographic determination of 3,4-dihydroxyphenylglycol, catecholamines, and 3,4-dihydroxyphenylalanine in plasma and their responses to inhibition of monoamine oxidase. Clin. Chem. 32:2030-2033.

26. Lenders, J.W.M., G. Eisenhofer, I. Armando, H.R. Keiser, D.S. Goldstein, and I.J. Kopin. 1993. Determination of metanephrines in plasma by liquid chromatography with electrochemical detection. Clin. Chem. 39:97-103.

27. Eisenhofer, G., W. Pecorella, K. Pacak, D. Hooper, I.J. Kopin, and D.S. Goldstein. 1994. The neuronal and extra-neuronal origins of plasma 3-methoxy4-hydroxyphenylglycol in rats. J. Auton. Nerv. Syst. 50:93-107.

28. Karoum, F. 1983. Mass fragmentography in the analysis of biogenic amines: a clinical, physiological, and pharmacological evaluation. In Methods in biogenic amine research. S. Parvez, T. Nagatsu, I. Nagatsu, and H. Parvez, editors. Elsevier, New York. 237-255.

29. Stroomer, A.E.M., H. Overmars, N.G.G.M. Abeling, and A.H. Van Gennip. 1990. Simultaneous detection of acidic 3,4-dihydroxyphenylalanine metabolites and 5-hydroxy-indole-3-acetic acid in urine by high-performance liquid chromatography. Clin. Chim. Acta. 36:1834-1837.

30. Abeling, N.G., A.H. van Gennip, H. Overmars, and P.A. Voûte. 1984 Simultaneous determination of catecholamines and metanephrines in urine by HPLC with fluorimetric detection. Clin. Chim. Acta. 137:211-226.

31. Karoum, F., H. Nasrallah, S. Potkin, L. Chuang, J. Moyer-Schwing, I. Phillips, and R.J. Wyatt. 1979. Mass fragmentography of phenylethylamine, $m$ - and $p$-tyramine, and related amines in plasma, cerebrospinal fluid, urine and brain. J. Neurochem. 33:201-202.

32. Pickar, D., R.M. Cohen, D.C. Jimerson, and D.L. Murphy. 1981. Tyramine infusions and selective monamine oxidase inhibitor treatment. I. Changes in pressor sensitivity. Psychopharmacology. 74:4-7.

33. Murphy, D.L., F. Karoum, I. Alterman, S. Lipper, and R.J. Wyatt. 1984. Phenylethylamine, tyramine and other trace amines in patients with affective disorders: associations with clinical state and antidepressant drug treatment. In Neurobiology of the trace amines. A.A. Boulton, G.B. Baker, W.G. Dewhurst, and M. Sandler, editors. Humana Press, Clifton, NJ. 449-514.

34. Glover, V., M. Sandler, F. Owen, and G.J. Riley. 1977. Dopamine is a monoamine oxidase B substrate in man. Nature (Lond.). 265:80-81.

35. Donnelly, C.H., and D.L. Murphy. 1977. Substrate- and inhibitorrelated characteristics of human platelet monoamine oxidase. Biochem. Pharmacol. 26:853-858.

36. Eisenhofer, G., D.S. Goldstein, T.G. Ropchak, and I.J. Kopin. 1988. Source and physiological significance of plasma 3,4-dihydroxyphenylalamine in the rat. J. Neurochem. 51:1204-1213. 
37. Buffoni, F. 1966. Histaminase and related amine oxidases. Pharmacol. Rev. 18:1163-1199.

38. McEwan, C.M. 1965. Human plasma monoamine oxidase. J. Biol. Chem. 240:2003-2010.

39. Campbell, I.C., D.S. Robinson, W. Lovenberg, and D.L. Murphy. 1979. The effects of chronic regimens of clorgyline and pargyline on monoamine oxidase metabolism in the rat brain. J. Neurochem. 32:49-55.

40. Murphy, D.L., N.A. Garrick, and R.M. Cohen. 1983. Monoamine oxidase inhibitors and monoamine oxidase: biochemical and physiological aspects relevant to human psycho-pharmacology. In Antidepressants. G.D. Burrows, T.R. Norman, and B. Davies, editors. Elsevier, Amsterdam. 209-227.

41. Buchsbaum, M.S., R.D. Coursey, and D.L. Murphy. 1976. The biochemical high-risk paradigm: behavioral and familial correlates of low-platelet monamine oxidase activity. Science (Wash. DC). 194:339-341.

42. von Knorring, L., L. Oreland, and B. Winblad. 1984. Personality traits related to monoamine oxidase activity in platelets. Psychiatr. Res. 12:11-26.

43. Devor, E.J., C.R. Cloninger, P.L. Hoffman, and B. Tabakoff. 1993. Association of monoamine oxidase (MAO) activity with alcoholism and alcoholic subtypes. Am. J. Med. Genet. 48:209-213.
44. Young, W.F., E. Laws, F.N. Sharbrough, and R.M. Weinshilbaum. 1986. Human monoamine oxidase, lack of brain and platelet correlation. Arch. Gen. Psychiatry. 43:604-609.

45. Chen, K., H.-F. Wu, and J.C. Shih. 1993. The deduced amino acid sequences of human platelet and frontal cortex monoamine oxidase B are identical. J. Neurochem. 61:187-190.

46. Paterson, I.A., A.V. Juorio, and A.A. Boulton. 1990. 2-Phenylethylamine: a modulator of catecholamine transmission in the mammalian central nervous system? J. Neurochem. 55:1827-1837.

47. Cases, O., I. Seif, J. Grimsby, P. Gaspar, K. Chen, S. Pournin, U. Müller, M. Aguet, C. Babinet, J. C. Shih, and E. De Maeyer. 1995. Aggressive behavior and altered amounts of brain serotonin and norepinephrine in mice lacking MAO-A. Science (Wash. DC). 268:1763-1766.

48. Whitaker-Azmitia, P.M., X. Zhang, and C. Clarke. 1994. Effects of gestational exposure to monoamine oxidase inhibitors in rats: preliminary behavioral and neurochemical studies. Neuropsychopharmacology. 11:125-132.

49. Brunner, H.G. 1995. MAOA deficiency and abnormal behaviour: perspectives on an association. In Genetics of Criminal and Antisocial Behaviour. M. Rutter, editor. Wily Liss, Chichester, UK. 155-167. 